\title{
THE HOMOTOPY THEORY OF POLYHEDRAL PRODUCTS ASSOCIATED WITH FLAG COMPLEXES
}

\author{
TARAS PANOV AND STEPHEN THERIAULT
}

\begin{abstract}
If $K$ is a simplicial complex on $m$ vertices the flagification of $K$ is the minimal flag complex $K^{f}$ on the same vertex set that contains $K$. Letting $L$ be the set of vertices, there is a sequence of simplicial inclusions $L \longrightarrow K \longrightarrow K^{f}$. This induces a sequence of maps of polyhedral products $(\underline{X}, \underline{A})^{L} \stackrel{g}{\longrightarrow}(\underline{X}, \underline{A})^{K} \stackrel{f}{\longrightarrow}(\underline{X}, \underline{A})^{K^{f}}$. We show that $\Omega f$ and $\Omega f \circ \Omega g$ have right homotopy inverses and draw consequences. For a flag complex $K$ the polyhedral product of the form $(\underline{C Y}, \underline{Y})^{K}$ is a co- $H$-space if and only if the 1-skeleton of $K$ is a chordal graph, and we deduce that the maps $f$ and $f \circ g$ have right homotopy inverses in this case.
\end{abstract}

\section{INTRODUCTION}

The purpose of this paper is to investigate the homotopy theory of polyhedral products associated with flag complexes. Polyhedral products have received considerable attention recently as they unify diverse constructions from several seemingly separate areas of mathematics: toric topology (moment-angle complexes), combinatorics (complements of complex coordinate subspace arrangements), commutative algebra (the Golod property of monomial rings), complex geometry (intersections of quadrics), and geometric group theory (Bestvina-Brady groups).

To be precise, let $K$ be a simplicial complex on the vertex set $[m]=\{1,2, \ldots, m\}$. For $1 \leq i \leq m$, let $\left(X_{i}, A_{i}\right)$ be a pair of pointed $C W$-complexes, where $A_{i}$ is a pointed $C W$-subcomplex of $X_{i}$. Let $(\underline{X}, \underline{A})=\left\{\left(X_{i}, A_{i}\right)\right\}_{i=1}^{m}$ be the sequence of pairs. For each simplex $\sigma \in K$, let $(\underline{X}, \underline{A})^{\sigma}$ be the subspace of $\prod_{i=1}^{m} X_{i}$ defined by

$$
(\underline{X}, \underline{A})^{\sigma}=\prod_{i=1}^{m} Y_{i} \quad \text { where } \quad Y_{i}=\left\{\begin{array}{cc}
X_{i} & \text { if } i \in \sigma \\
A_{i} & \text { if } i \notin \sigma .
\end{array}\right.
$$

The polyhedral product determined by $(\underline{X}, \underline{A})$ and $K$ is

$$
(\underline{X}, \underline{A})^{K}=\bigcup_{\sigma \in K}(\underline{X}, \underline{A})^{\sigma} \subseteq \prod_{i=1}^{m} X_{i} .
$$

For example, suppose each $A_{i}$ is a point. If $K$ is a disjoint union of $m$ points then $(\underline{X}, \underline{*})^{K}$ is the wedge $X_{1} \vee \cdots \vee X_{m}$, and if $K$ is the standard $(m-1)$-simplex then $\left(\underline{X},{ }^{*}\right)^{K}$ is the product $X_{1} \times \cdots \times X_{m}$.

The combinatorics of $K$ informs greatly on the homotopy theory of $(\underline{X}, \underline{A})^{K}$. One notable family of simplicial complexes is the collection of flag complexes. A simplicial complex $K$ is flag if any set of

2010 Mathematics Subject Classification. Primary 55P35, Secondary 05 E45.

Key words and phrases. polyhedral product, moment-angle complex, flag complex. 
vertices of $K$ which are pairwise connected by edges spans a simplex. Flag complexes are important in graph theory, where they are referred to as clique complexes, in the study of metric spaces, where they are referred to as Rips complexes, and in geometric group theory, where they are referred to as Gromov's no- $\triangle$ complexes.

The flagification of $K$, denoted $K^{f}$, is the minimal flag complex on the same set $[m]$ that contains $K$. We therefore have a simplicial inclusion $K \rightarrow K^{f}$. For example, the $(m-1)$-simplex $\Delta^{m-1}$, consisting of all subsets of $[m]$, is flag, while its boundary $\partial \Delta^{m-1}$, consisting of all proper subsets of $[m]$, is flag only for $m=2$. The flagification of $\partial \Delta^{m-1}$ with $m>2$ is $\Delta^{m-1}$. An $m$-cycle (the boundary of an $m$-gon) is flag whenever $m>3$.

The main result of the paper is the following.

Theorem 1.1. Let $K$ be a simplicial complex on the vertex set $[m]$, let $K^{f}$ be the flagification of $K$, and let $L$ be the simplicial complex given by $m$ disjoint points. Let $(\underline{X}, \underline{A})=\left\{\left(X_{i}, A_{i}\right)\right\}_{i=1}^{m}$ be a sequence of pairs of pointed $C W$-complexes, where $A_{i}$ is a pointed $C W$-subcomplex of $X_{i}$. Let $(\underline{X}, \underline{A})^{L} \stackrel{g}{\longrightarrow}(\underline{X}, \underline{A})^{K} \stackrel{f}{\longrightarrow}(\underline{X}, \underline{A})^{K^{f}}$ be the maps of polyhedral products induced by the maps of simplicial complexes $L \longrightarrow K \longrightarrow K^{f}$. Then the following hold:

(a) the map $\Omega f$ has a right homotopy inverse;

(b) the composite $\Omega f \circ \Omega g$ has a right homotopy inverse.

In particular, consider the special case when each $A_{i}$ is a point. Write $(\underline{X}, \underline{*})$ for $(\underline{X}, \underline{A})$ and notice that $(\underline{X}, \underline{*})^{L}=X_{1} \vee \cdots \vee X_{m}$. If $K$ is a flag complex on the vertex set $[m]$ then the simplicial map $L \longrightarrow K$ induces a map $f: X_{1} \vee \cdots \vee X_{m}=(\underline{X}, \underline{*})^{L} \longrightarrow(\underline{X}, \underline{*})^{K}$. By Theorem $1.1, \Omega f$ has a right homotopy inverse. That is, $\Omega(\underline{X}, \underline{*})^{K}$ is a retract of $\Omega\left(X_{1} \vee \cdots \vee X_{m}\right)$. This informs greatly on the homotopy theory of $\Omega(\underline{X}, \underline{*})^{K}$ since the homotopy type of $\Omega\left(X_{1} \vee \cdots \vee X_{m}\right)$ has been well studied; in particular, in the special case when each $X_{i}$ is a suspension the Hilton-Milnor Theorem gives an explicit homotopy decomposition of the loops on the wedge. Theorem 1.1 also greatly generalizes [GPTW, Theorem 5.3], which stated that such a retraction exists in the special case when each $X_{i}=\mathbb{C} P^{\infty}$ provided spaces and maps have been localized at a prime $p \neq 2$.

Theorem 1.1 can be improved in certain cases. In Section 6 we consider polyhedral products of the form $(\underline{C Y}, \underline{Y})^{K}$, where $C Y$ is the cone on $Y$, and identify the class of flag complexes $K$ for which $(\underline{C Y}, \underline{Y})^{K}$ is a co- $H$-space. As a corollary, we obtain conditions that allow for a delooping of the statement of Theorem 1.1. In Section 7 we relate Theorem 1.1 to Whitehead products. First, we consider polyhedral products of the form $(\underline{X}, \underline{*})^{K}$ with flag $K$ whose 1-skeleton is a chordal graph, and obtain a generalisation of Porter's description of the homotopy fiber of the inclusion of an $m$-fold wedge into a product in terms of Whitehead brackets. Second, we consider the loop space $\Omega(\underline{S}, \underline{*})^{K}$ on a polyhedral product formed from spheres for an arbitrary flag complex $K$, and obtain a generalisation of the Hilton-Milnor Theorem.

The research of the first author was carried out at the Institute for Information Transmission Problems of Russian Academy of Sciences and was supported by the Russian Science Foundation 
(grant no. 14-50-00150). The second author would like to thank the Royal Society for the award of an International Exchanges Grant which helped make this research possible. The authors would also like to thank the referee for making several helpful comments.

\section{Combinatorial PREPARATion}

This section records the combinatorial information that will be needed. We begin with some definitions. Let $K$ be an abstract simplicial complex on the set $[m]=\{1,2, \ldots, m\}$, i.e. $K$ is a collection of subsets $\sigma \subseteq[m]$ such that for any $\sigma \in K$ all subsets of $\sigma$ also belong to $K$. We refer to $\sigma \in K$ as a simplex (or a face) of $K$ and denote by $|\sigma|$ the number of elements in $\sigma$. We always assume that the empty set $\varnothing$ belongs to $K$. We do not assume that $K$ contains all one-element subsets $\{i\} \subseteq[m]$. We refer to $\{i\} \in K$ as a vertex of $K$, and refer to $\{i\} \notin K$ as a ghost vertex. We say that $K$ is a simplicial complex on the vertex set $[m]$ when there are no ghost vertices.

Let $K$ be a simplicial complex on the set $[m]$. For a vertex $v \in K$, the star, restriction (or deletion) and link of $v$ are the subcomplexes

$$
\begin{aligned}
\operatorname{star}_{K}(v) & =\{\tau \in K \mid\{v\} \cup \tau \in K\} ; \\
K \backslash v & =\{\tau \in K \mid\{v\} \cap \tau=\varnothing\} ; \\
\operatorname{link}_{K}(v) & =\operatorname{star}_{K}(v) \cap K \backslash v .
\end{aligned}
$$

Throughout the paper we follow the convention of regarding $\operatorname{star}_{K}(v)$ as a simplicial complex on the same set $[m]$ as $K$, while regarding $K \backslash v$ and $\operatorname{link}_{K} v$ as simplicial complexes on the set $[m] \backslash v$. This implies that $\operatorname{star}_{K}(v)$ and $\operatorname{link}_{K} v$ may have ghost vertices even if $K$ does not.

The join of two simplicial complexes $K_{1}, K_{2}$ on disjoint sets is the simplicial complex

$$
K_{1} * K_{2}=\left\{\sigma_{1} \cup \sigma_{2} \mid \sigma_{i} \in K_{i}\right\}
$$

From the definitions, it follows that $\operatorname{star}_{K}(v)$ is a join,

$$
\operatorname{star}_{K}(v)=\{v\} * \operatorname{link}_{K}(v),
$$

and there is a pushout

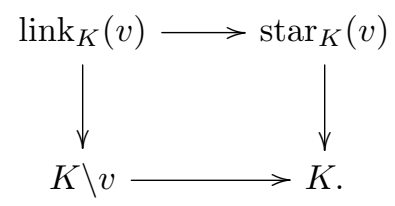

A non-face of $K$ is a subset $\omega \subseteq[m]$ such that $\omega \notin K$. A missing face (a minimal non-face) of $K$ is an inclusion-minimal non-face of $K$, that is, a subset $\omega \subseteq[m]$ such that $\omega$ is not a simplex of $K$, but every proper subset of $\omega$ is a simplex of $K$. A ghost vertex is therefore a missing face consisting of one element. Denote the set of missing faces of $K$ by $\operatorname{MF}(K)$. For a subset $\omega \subseteq[m]$, let $\partial \omega$ denote the collection of proper subsets of $\omega$. Observe that $\omega \in \operatorname{MF}(K)$ if and only if $\omega \notin K$ but $\partial \omega \subseteq K$.

A simplicial complex $K$ on the set $[m]$ is called a flag complex if each of its missing faces consists of at most two elements. Equivalently, $K$ is flag if any set of vertices of $K$ which are pairwise 
connected by edges spans a simplex. Every flag complex $K$ is determined by its 1-skeleton $K^{1}$, and is obtained from the graph $K^{1}$ by filling in all cliques (complete subgraphs) by simplices.

Lemma 2.1. Let $K$ be a flag complex on the set $[m]$ and let $v$ be a vertex of $K$. If $\omega \in \operatorname{MF}\left(\operatorname{link}_{K}(v)\right)$ and $|\omega| \geq 2$, then $\omega \in \operatorname{MF}(K \backslash\{v\})$.

Proof. Suppose not. Then there is a missing face $\omega$ of $\operatorname{link}_{K}(v)$ with $\omega \in K \backslash\{v\}$ and $|\omega| \geq 2$. Therefore, $\partial \omega \subseteq \operatorname{link}_{K}(v)$ but $\omega \notin \operatorname{link}_{K}(v)$. Since $\omega \in K \backslash\{v\}$, we also have $\omega \in K$. On the other

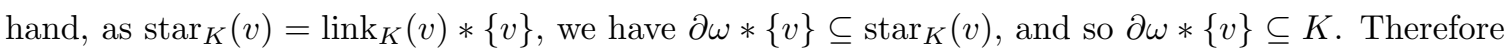
$\partial \omega *\{v\} \cup \omega \subseteq K$.

Observe that $\partial \omega *\{v\} \cup \omega=\partial \tau$ where $\tau=\omega *\{v\}$. Thus $\partial \tau \subseteq K$. As $K$ is flag and $|\omega *\{v\}|>2$, this implies that $\tau=\omega *\{v\} \in K$. Hence, $\omega \in \operatorname{link}_{K} v$, a contradiction.

Lemma 2.2. Let $K$ be a flag complex on the set $[m]$ and let $v$ be a vertex of $K$. Then $K \backslash\{v\}$, $\operatorname{star}_{K}(v)$ and $\operatorname{link}_{K}(v)$ are all flag complexes.

Proof. Since $K \backslash\{v\}$ is a full subcomplex of $K$, any missing face of $K \backslash\{v\}$ is also a missing face of $K$. So as $K$ is flag, any missing face has at most two elements, implying that any missing face of $K \backslash\{v\}$ also has at most two elements. Thus $K \backslash\{v\}$ is flag.

Let $\omega \in \operatorname{MF}\left(\operatorname{star}_{K}(v)\right)$ and $|\omega| \geq 2$. We claim that $\omega \in \operatorname{MF}(K)$ as well. As $\partial \omega \subseteq \operatorname{star}_{K}(v)$, we also have $\partial \omega \subseteq K$, so if the claim does not hold then it must be the case that $\omega \in K$. Then $v \notin \omega$, as otherwise $\omega \in \operatorname{star}_{K}(v)$. For $\tau=\omega * v$ we have $\partial \tau=\partial \omega * v \cup \omega \in K$. As $K$ is flag and $|\omega *\{v\}|>2$, we obtain $\tau=\omega *\{v\} \in K$. This implies that $\omega \in \operatorname{star}_{K}(v)$, a contradiction. Hence, $\omega \in \operatorname{MF}(K)$

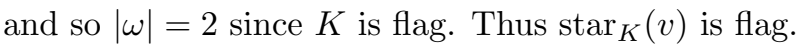

Let $\omega \in \operatorname{MF}\left(\operatorname{link}_{K}(v)\right)$ and $|\omega| \geq 2$. By Lemma 2.1, $\omega \in \operatorname{MF}(K \backslash\{v\})$ as well. It has already been established that $K \backslash\{v\}$ is flag, so we have $|\omega|=2$. Thus $\operatorname{link}_{K}(v)$ is also flag.

Given a subset $\omega \subseteq[m]$, the full subcomplex of $K$ on $\omega$ is

$$
K_{\omega}=\{\sigma \in K \mid \sigma \subseteq \omega\}
$$

Note that $K \backslash\{v\}=K_{[m] \backslash\{v\}}$. A key property that will be important subsequently is the following.

Lemma 2.3. Let $K$ be a flag complex on the set $[m]$ and let $v$ be a vertex of $K$. Then $\operatorname{link}_{K}(v)$ is a full subcomplex of $K \backslash\{v\}$.

Proof. Let $\omega$ be the vertex set of $\operatorname{link}_{K}(v)$. Suppose that $\operatorname{link}_{K}(v)$ is not a full subcomplex of $K \backslash\{v\}$. Then there is a face $\sigma \in K \backslash\{v\}$ such that $\sigma \subseteq \omega$ and $\sigma \notin \operatorname{link}_{K}(v)$. By selecting a proper face of $\sigma$ if necessary, we may assume that $\sigma$ is a missing face of $\operatorname{link}_{K}(v)$ with $|\sigma| \geq 2$. But then as $K$ is flag, Lemma 2.1 implies that $\sigma$ is also a missing face of $K \backslash\{v\}$. In particular, $\sigma \notin K \backslash\{v\}$, a contradiction. 


\section{Homotopy theORETIC PREPARATION}

3.1. The Cube Lemma. Assume that all spaces are pointed and have nondegenerate basepoints, implying that the inclusion of the basepoint is a cofibration. This holds, for example, for pointed $C W$-complexes, and hence for polyhedral products. One part of Mather's Cube Lemma [Ma] states that if there is a diagram of spaces and maps

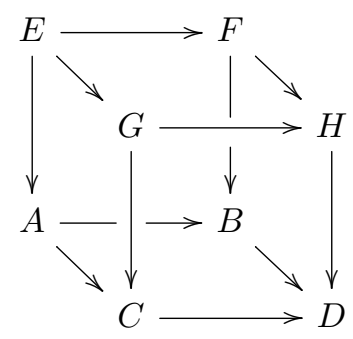

where the bottom face is a homotopy pushout and the four sides are obtained by pulling back with $H \longrightarrow D$, then the top face is also a homotopy pushout. In what follows this will be used to identify the homotopy type of the pushout $H$ in a certain context. However, we need this identification to have a naturality property, which is not immediate from the statement of the Cube Lemma. To obtain this, we prove a special case of the Cube Lemma from first principles.

In what follows, we work with strictly commutative pushouts and pullbacks rather than homotopy commutative ones. For a space $Y$ let $1_{Y}$ be the identity map on $Y$. Suppose that there is a strictly commutative diagram

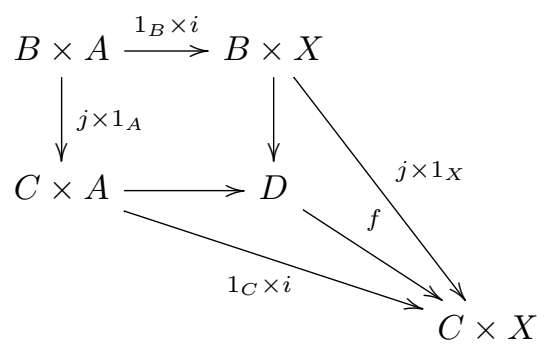

where the square is a pushout, and the maps $i, j$ and $f$ are pointed inclusions of subspaces. We will turn the maps $f, 1_{C} \times i, j \times 1_{X}$ and $j \times i$ from the four corners of the pushout to $C \times X$ into fibrations, up to homotopy, and examine their fibres.

There is a standard way of turning a pointed, continuous map $g: Y \longrightarrow Z$ between locally compact, Hausdorff spaces into a fibration, up to homotopy. Let $I$ be the unit interval and let $\operatorname{Map}(I, Z)$ be the space of continuous (not necessarily pointed) maps from $I$ to $Z$. Let $d$ : $\operatorname{Map}(I, Z) \longrightarrow Z \times Z$ be defined by evaluating a map $\omega: I \longrightarrow Z$ at the two endpoints, explicitly, $d(\omega)=(\omega(0), \omega(1))$. Define the space $\widetilde{P}_{g}$ by the pullback

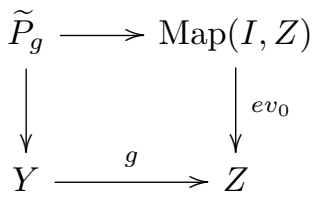


where $e v_{0}(\omega)=\omega(0)$. As a set,

$$
\widetilde{P}_{g}=\{(y, \omega) \in Y \times \operatorname{Map}(I, Z) \mid \omega(0)=g(y)\} .
$$

Then, as in [Se, p. 59] for example, there is an inclusion $Y \longrightarrow \widetilde{P}_{g}$ which is a homotopy equivalence and the composite

$$
q: \widetilde{P}_{g} \longrightarrow \operatorname{Map}(I, Z) \stackrel{e v_{1}}{\longrightarrow} Z
$$

is a fibration, where $e v_{1}(\omega)=\omega(1)$. Moreover, if 1 is the basepoint of $I$ and $P Z$ is the path space of $Z$ (with paths at time 1 ending at the basepoint of $Z$ ), then the fibre of $q$ is homeomorphic to the mapping path space of $g$,

$$
P_{g}=\{(y, \omega) \in Y \times P Z \mid \omega(0)=g(y)\},
$$

which is obtained by the pullback

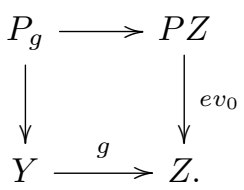

Consider how these constructions behave with respect to pointed subspace inclusions. Let $S \stackrel{s}{\longrightarrow} Y$ be the inclusion of a pointed subspace. If $Q$ is the pullback of $S \stackrel{s}{\longrightarrow} Y$ and $\widetilde{P}_{g} \longrightarrow Y$, then the pullback defining $\widetilde{P}_{g}$ implies that $Q$ is also the pullback of $g \circ s$ and $e v_{0}$. But this pullback is the definition of $\widetilde{P}_{g \circ s}$, so $Q=\widetilde{P}_{g \circ s}$. Similarly for $P_{g \circ s}$, giving pullbacks
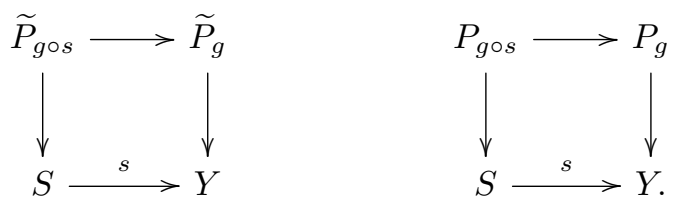

Since $P_{g}$ and $P_{g \circ s}$ are the respective fibres of $\widetilde{P}_{g}$ and $\widetilde{P}_{g \circ s}$ over $Z$, we obtain a pullback

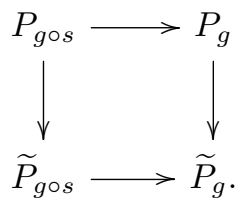

Next, suppose that $Y$ is the union of pointed, closed subspaces $S$ and $T$. Let $s: S \longrightarrow Y$ and $t: T \longrightarrow Y$ be the pointed subspace inclusions, and let $u$ and $v$ be the pointed subspace inclusions $u: S \cap T \longrightarrow S$ and $v: S \cap T \longrightarrow T$. Since $S$ and $T$ are closed subspaces of $Y$, the pushout of $u$ and $v$ is $Y$. (More generally this is true if $(Y ; S, T)$ is an excisive triad, but we do not need this level of generality - in our case each of $S, T$ and $Y$ will be certain polyhedral products.) 
Lemma 3.1. Suppose that $Y \stackrel{g}{\longrightarrow} Z$ is a pointed subspace inclusion and that $Y=S \cup T$ where $S$ and $T$ are closed, pointed subspaces of $Y$ Then there are pushouts
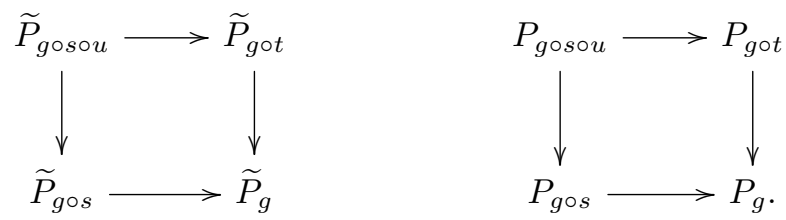

Proof. By its definition, $\widetilde{P}_{g}$ is the space of paths on $Z$ that begin in $\operatorname{Im}(g)$ and end in $Z$. As $g$ is a subspace inclusion, we may regard $\widetilde{P}_{g}$ as the space of paths on $Z$ that begin in $Y$ and end in $Z$. As $Y=S \cup T$, any such path either begins in $S$ or in $T$ - that is - the path is either in $\widetilde{P}_{g \circ s}$ or $\widetilde{P}_{g \circ t}$. Moreover, the intersection $\widetilde{P}_{g \circ s} \cap \widetilde{P}_{g \circ t}$ is all paths on $Z$ that begin in $S \cap T$ and end in $Z$ - that is - the paths in $\widetilde{P}_{g \circ s \circ u}=\widetilde{P}_{g \circ t o v}$. Thus $\widetilde{P}_{g}=\widetilde{P}_{g \circ s} \cup \widetilde{P}_{g \circ t}$ and $\widetilde{P}_{g \circ s \circ u}=\widetilde{P}_{g \circ s} \cap \widetilde{P}_{g \circ t}$. Further, since $S$ and $T$ are closed subspaces of $Y$, we have $\widetilde{P}_{g \circ s}$ and $\widetilde{P}_{g \circ t}$ closed subspaces of $\widetilde{P}_{g}$. Therefore there is a pushout

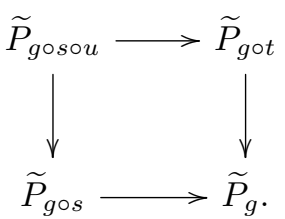

The same argument shows that $P_{g}$ is the pushout of $P_{g \circ s}$ and $P_{g \circ t}$ over $P_{g \circ s \circ u}=P_{g \circ t o v}$.

Now apply this construction to the maps $f, 1_{C} \times i, j \times 1_{X}$ and $j \times i$ from the four corners of the pushout in (2) to $C \times X$.

Lemma 3.2. There is a commutative cube

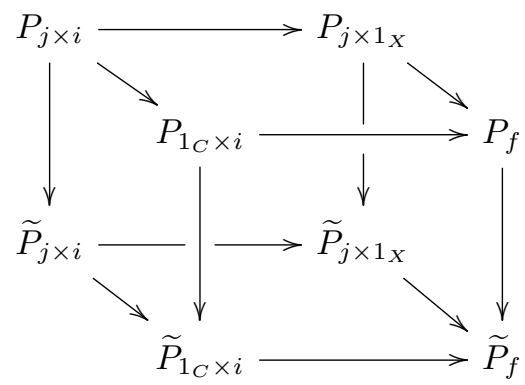

where the top and bottom faces are pushouts and the four sides are pullbacks. Further, this cube is natural for maps of diagrams of the form (2).

Proof. Since $f, 1_{C} \times i, j \times 1_{X}$ and $j \times i$ are all subspace inclusons, the four sides of the cube are pullbacks by (5). Since $D$ is a pushout, it is the union of $C \times A$ and $B \times X$ with intersection $B \times A$. The top and bottom faces of the cube are therefore pushouts by (6). The naturality statement holds since the constructions of $\widetilde{P}_{g}$ and $P_{g}$ are natural.

The top face of the cube in Lemma 3.2 will be more precisely identified. This requires two lemmas. 
Lemma 3.3. A map $g \times h: Y \times M \longrightarrow Z \times N$ has $P_{g \times h}=P_{g} \times P_{h}$. Further, this decomposition is natural for compositions $s \times t: Z \times N \longrightarrow Z^{\prime} \times N^{\prime}$.

Proof. First observe that $P(Z \times N)=P Z \times P N$ since any pointed path $\omega: I \longrightarrow Z \times N$ is equivalent to the product of the pointed paths $\omega_{1}: I \longrightarrow Z$ and $\omega_{2}: I \longrightarrow N$ given by projecting $\omega$ to $Z$ and $N$ respectively. Moreover, the evaluation map $P(Z \times N) \stackrel{e v_{0}}{\longrightarrow} Z \times N$ becomes a product of evaluation maps $P Z \times P N \stackrel{e v_{0} \times e v_{0}}{\longrightarrow} Z \times N$. Thus the pullback $P_{g \times h}$ is identical to the pullback

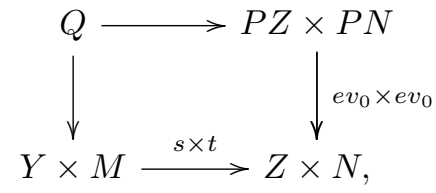

where

$$
\begin{aligned}
Q & =\left\{\left((y, m),\left(\omega_{1}, \omega_{2}\right) \in Y \times M \times P Z \times P N \mid s(y)=\omega_{1}(0), t(m)=\omega_{2}(0)\right\}\right. \\
& =\left\{\left(y, \omega_{1}\right) \in Y \times P Z \mid s(y)=\omega_{1}(0)\right\} \times\left\{\left(m, \omega_{2}\right) \in M \times P N \mid t(m)=\omega_{2}(0)\right\} \\
& =P_{s} \times P_{t} .
\end{aligned}
$$

The identification of $P_{s \times t}$ as $P_{s} \times P_{t}$ only used the fact that $P(Z \times N)=P Z \times P N$. As the latter decomposition is natural, therefore so is the former.

Lemma 3.4. There is a natural homeomorphism $P_{1_{Y}} \cong P Y$.

Proof. Taking $g=1_{Y}$ in (4) gives

$$
P_{1_{Y}}=\{(y, \omega) \in Y \times P Y \mid \omega(0)=y\} .
$$

Define $\phi: P Y \longrightarrow P_{1_{Y}}$ by $\phi(\omega)=(\omega(0), \omega)$ and $\psi: P_{1_{Y}} \longrightarrow P Y$ by $\psi(y, \omega)=\omega$. Both $\phi$ and $\psi$ are continuous, $\psi \circ \phi=i d_{P Y}$ and, because for any pair $(y, \omega) \in P_{1_{Y}}$ there is the condition $y=\omega(0)$, we also have $\phi \circ \psi=1_{P_{1_{Y}}}$. Hence $\psi$ is a homeomorphism. As both $\phi$ and $\psi$ are natural, the homeomorphism is too.

Applying Lemmas 3.3 and 3.4 to the top face in Lemma 3.2, the space $P_{f}$ is homeomorphic to the space $Q_{f}$ defined by the pushout

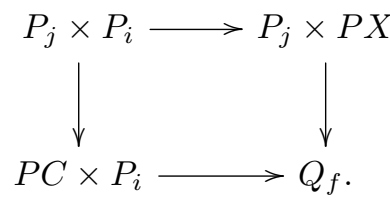

Moreover, the naturality statements in Lemmas 3.2 through 3.4 imply that (7) is natural for maps of diagrams of the form (2).

One further modification of (7) is needed. If $Y$ is a pointed space the reduced cone on $Y$ is the space $C Y=Y \wedge I$ (i.e., $C Y=(Y \times I) /(Y \vee I))$. If $Y$ and $Z$ are pointed spaces with basepoints $y_{0}$ and $z_{0}$ respectively, then the reduced join is defined by $Y * Z=(Y \times I \times Z) / \sim$, where $(y, 0, z)=\left(y, 0, z^{\prime}\right)$, 
$(y, 1, z) \sim\left(y^{\prime}, 1, z\right)$ and $\left(y_{0}, t, z_{0}\right)=\left(y_{0}, 0, z_{0}\right)$ for all $y, y^{\prime} \in Y, z, z^{\prime} \in Z$ and $t \in I$. Observe that there is a pushout

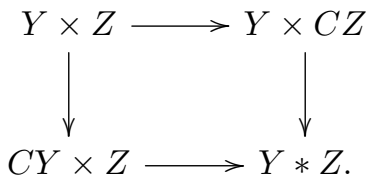

Proposition 3.5. Up to homotopy equivalences, the top face in Lemma 3.2 can be identified with the pushout

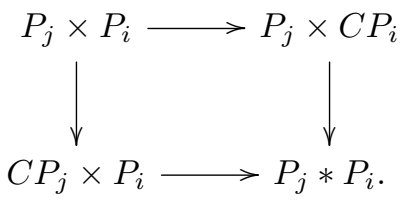

In particular, $P_{f}$ is homotopy equivalent to $P_{j} * P_{i}$. Further, this homotopy equivalence may be chosen to be natural for maps of diagrams of the form (2).

Proof. In general, suppose that $Z$ is contractible. Then there is a pointed homotopy $Z \times I \longrightarrow Z$ which at $t=0$ is the identity map on $Z$ and at $t=1$ is the constant map to the basepoint. The homotopy sends $Z \vee I$ to the basepoint, and so factors through a map $C Z=Z \wedge I \longrightarrow Z$. That is, the contracting homotopy for $Z$ determines a specific map $C Z \longrightarrow Z$. If the contracting homotopy is natural for maps $Z \longrightarrow Z^{\prime}$, then the map $C Z \longrightarrow Z$ is also natural. In fact, it is a natural homotopy equivalence. Refining, if $g: Y \longrightarrow Z$ is a pointed map with $Z$ being contractible, then we obtain a composite $C Y \stackrel{C g}{\longrightarrow} C Z \longrightarrow Z$ with the same naturality properties.

In our case, consider (7). Since $P C$ and $P X$ are contractible, we obtain composites $P_{j} \longrightarrow$ $C P_{j} \longrightarrow P C$ and $P_{i} \longrightarrow C P_{i} \longrightarrow P X$ in which the right hand maps are homotopy equivalences. Thus the pushout $Q_{f}$ in (7) is homotopy equivalent to the space $P_{j} * P_{i}$ obtained from the pushout

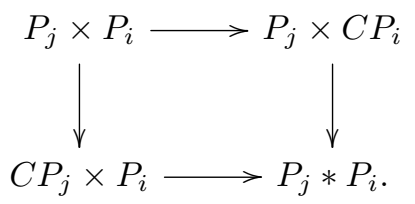

Since $P_{f}$ is homeomorphic to $Q_{f}$, we obtain $P_{f} \simeq P_{j} * P_{i}$. Further, since the contracting homotopy for a path space $P Z$ can be chosen to be natural for any map $Z \longrightarrow Z^{\prime}$, this homotopy equivalence for $P_{f}$ is natural to the same extent as $(7)$ is natural. That is, it is natural for maps of diagrams of the form (2).

3.2. Two general results on fibrations. Now assume that all spaces have the homotopy type of pointed $C W$-complexes. If $X$ is such a space then by [Mi, Corollary 3] so is $\Omega X$. Also, any weak homotopy equivalence between two such spaces is a homotopy equivalence (see, for example, [Sp, Ch. 7, §6, Corollary 24].

Lemma 3.6. Suppose that $\Omega B \stackrel{\partial}{\longrightarrow} F \stackrel{f}{\longrightarrow} E \stackrel{p}{\longrightarrow} B$ is a homotopy fibration sequence and $p$ has a left homotopy inverse. Then $\partial$ has a right homotopy inverse. 
Proof. Let $s: B \longrightarrow E$ be a map such that $s \circ p$ is homotopic to the identity map on $E$. Then $f \simeq$ $s \circ p \circ f$, implying that $f$ is null homotopic since $p \circ f$ is. If $X$ is any pointed space then the homotopy fibration $\Omega B \stackrel{\partial}{\longrightarrow} F \stackrel{f}{\longrightarrow} E$ induces an exact sequence of pointed sets $[X, \Omega B] \stackrel{\partial_{*}}{\longrightarrow}[X, F] \stackrel{f_{*}}{\longrightarrow}[X, B]$ where $[X, Y]$ is the set of pointed homotopy classes of maps from $X$ to $Y$. Since $f$ is null homotopic, $f_{*}=0$, so $\partial_{*}$ is onto. Taking $X=F$ implies that the (homotopy class of the) identity map on $F$ lifts through $\partial_{*}$ to a map $t: F \longrightarrow \Omega B$. That is, $\partial \circ t$ is homotopic to the identity map on $F$.

In general, if $F \stackrel{f}{\longrightarrow} E \stackrel{p}{\longrightarrow} B$ is a homotopy fibration where $E$ is an $H$-space and $p$ has a right homotopy inverse $s: B \longrightarrow E$, then the composite

$$
B \times F \stackrel{s \times f}{\longrightarrow} E \times E \stackrel{\mu}{\longrightarrow} E
$$

is a weak homotopy equivalence, and hence a homotopy equivalence. We wish to give a slight variation on this in the case when $B=B_{1} \times B_{2}$ and each factor has a right homotopy inverse. For $i=1,2$ let $p_{i}$ be the composite $p_{i}: E \stackrel{p}{\longrightarrow} B_{1} \times B_{2} \stackrel{\pi_{i}}{\longrightarrow} B_{i}$ where $\pi_{i}$ is the projection. As maps into a product are determined by their projection onto each factor, we have $p=\left(p_{1}, p_{2}\right)$.

Lemma 3.7. Let $F \stackrel{f}{\longrightarrow} E \stackrel{p}{\longrightarrow} B_{1} \times B_{2}$ be a homotopy fibration where $p$ is an $H$-map. Suppose that for $i=1,2$ there are maps $s_{i}: B_{i} \longrightarrow E$ such that $p_{i} \circ s_{i}$ is homotopic to the identity map on $B_{i}$, and $p_{i} \circ s_{j}$ is null homotopic for $i \neq j$. Then the composite

$$
B_{1} \times B_{2} \times F \stackrel{s_{1} \times s_{2} \times f}{\longrightarrow} E \times E \times E \stackrel{\mu \circ(\mu \times 1)}{\longrightarrow} E
$$

is a homotopy equivalence, where $\mu$ is the multiplication on $E$.

Proof. From the general result stated before the lemma, it suffices to show that $s_{1} \times s_{2}$ is a right homotopy inverse for $p$. Consider the diagram

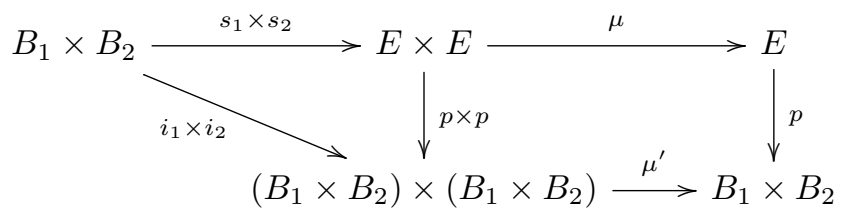

where $i_{1}$ and $i_{2}$ are the inclusions into the first and second factors respectively and $\mu^{\prime}$ is the multiplication on $B_{1} \times B_{2}$. The left triangle homotopy commutes since $p_{i} \circ s_{i}$ is homotopic to the identity map on $B_{i}$ and $p_{i} \circ s_{j} \simeq *$ if $i \neq j$. The right square homotopy commutes since $p$ is an $H$-map. Observe that the lower direction around the diagram is homotopic to the identity map on $B_{1} \times B_{2}$. Therefore the upper direction around the diagram implies that $\mu \circ\left(s_{1} \times s_{2}\right)$ is a right homotopy inverse for $p$.

\section{Polyhedral products and the Proof of Theorem 1.1}

Let $K$ be a simplicial complex on the set $[m]$ and let $v$ be a vertex of $K$. Following Félix and Tanré $[\mathrm{FT}]$, define a new simplicial complex $\bar{K}$ on $[m]$ by

$$
\bar{K}=K \backslash\{v\} *\{v\} .
$$


Observe that there is an inclusion of simplicial complexes $K \backslash\{v\} \longrightarrow \bar{K}$ given by including the join factor, so as $\operatorname{star}_{K}(v)=\operatorname{link}_{K}(v) *\{v\}$, there is a pushout map

$$
K \longrightarrow \bar{K}
$$

Observe also that $K \backslash\{v\}$ is the full subcomplex of $\bar{K}$. That is, $\bar{K} \backslash\{v\}=K \backslash\{v\}$.

By [GT2], the pushout of simplicial complexes in (1) induces a pushout of polyhedral products

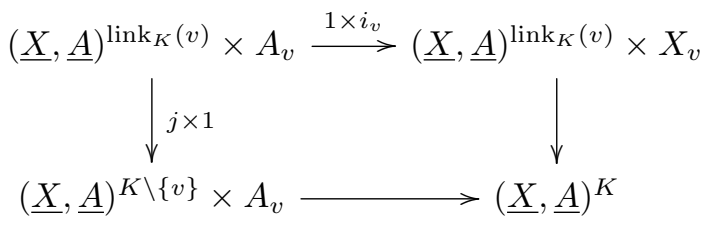

where $i_{v}$ is the inclusion. (Here we regard $\operatorname{link}_{K}(v)$ and $K \backslash\{v\}$ as simplicial complexes on the set $[m] \backslash\{v\}$.) To relate this to $(\underline{X}, \underline{A})^{\bar{K}}$, observe that the definition of the join of two simplicial complexes implies that if $K=K_{1} * K_{2}$ then there is a homeomorphism

$$
(\underline{X}, \underline{A})^{K} \cong(\underline{X}, \underline{A})^{K_{1}} \times(\underline{X}, \underline{A})^{K_{2}} \text {. }
$$

In particular, as $\bar{K}=K \backslash\{v\} *\{v\}$ there is a homeomorphism

$$
(\underline{X}, \underline{A})^{\bar{K}} \cong(\underline{X}, \underline{A})^{K \backslash\{v\}} \times X_{v}
$$

and a strictly commutative diagram

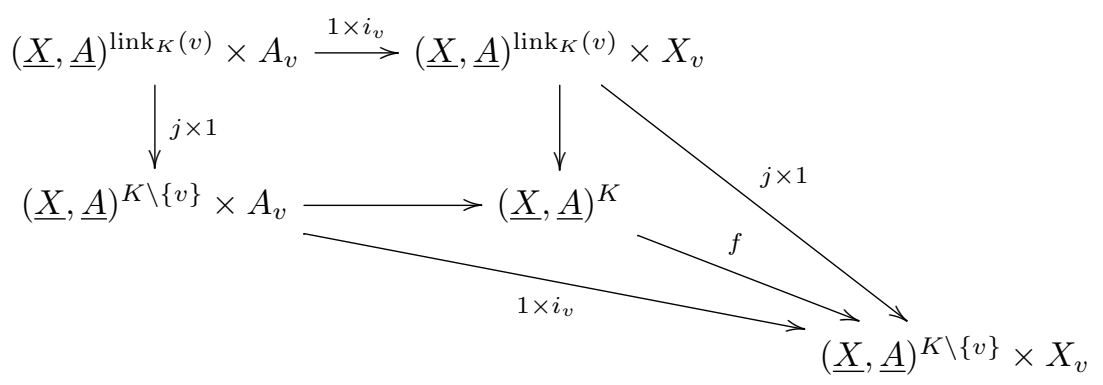

where $f$ is the map induced by the simplicial map $K \longrightarrow \bar{K}$ and all maps are inclusions of subspaces.

Let $B_{v}^{K}$ be the fibre $P_{j}$ obtained by turning the map $(\underline{X}, \underline{A})^{\operatorname{link}_{K}(v)} \stackrel{j}{\longrightarrow}(\underline{X}, \underline{A})^{K \backslash\{v\}}$ into a fibration and let $Y_{v}$ be the fibre $P_{i_{v}}$ obtained by turning the inclusion $A_{v} \stackrel{i_{v}}{\longrightarrow} X_{v}$ into a fibration.

Lemma 4.1. If $F_{v}^{K}$ is the fibre $P_{f}$ obtained by turning the $\operatorname{map}(\underline{X}, \underline{A})^{K} \stackrel{f}{\longrightarrow}(\underline{X}, \underline{A})^{K \backslash\{v\}} \times X_{v}$ into a fibration, then there is a homotopy equivalence

$$
F_{v}^{K} \simeq B_{v}^{K} * Y_{v} .
$$

Further, this homotopy equivalence is natural for inclusions of simplicial complexes $K \longrightarrow K^{\prime}$ on the set $[m]$.

Proof. Proposition 3.5 immediately implies the asserted homotopy equivalence for $F_{v}^{K}$ and states that it is natural for maps of diagrams of the form (9). Now observe that any inclusion of simplicial complexes $K \longrightarrow K^{\prime}$ on the vertex set $[m]$ induces such a map of diagrams. 
To take this further we need a general result about polyhedral products.

Lemma 4.2. Suppose that $K_{\omega}$ is a full subcomplex of a simplicial complex $K$. Then the map of polyhedral products $(\underline{X}, \underline{A})^{K_{\omega}} \longrightarrow(\underline{X}, \underline{A})^{K}$ induced by the simplicial inclusion $K_{\omega} \longrightarrow K$ has a left inverse, that is, there is a retraction $(\underline{X}, \underline{A})^{K} \longrightarrow(\underline{X}, \underline{A})^{K_{\omega}}$. Further, the construction of the left inverse is natural for simplicial inclusions $K \longrightarrow K^{\prime}$.

Proof. We have

$$
(\underline{X}, \underline{A})^{K}=\bigcup_{\sigma \in \mathcal{K}}\left(\prod_{i \in \sigma} X_{i} \times \prod_{i \in[m] \backslash \sigma} A_{i}\right), \quad(\underline{X}, \underline{A})^{K_{\omega}}=\bigcup_{\sigma \in \mathcal{K}, \sigma \subseteq \omega}\left(\prod_{i \in \sigma} X_{i} \times \prod_{i \in \omega \backslash \sigma} A_{i}\right) .
$$

Since each $A_{i}$ is a pointed space, there is a canonical inclusion $(\underline{X}, \underline{A})^{K_{\omega}} \longrightarrow(\underline{X}, \underline{A})^{K}$. Furthermore, for each $\sigma \in K$ there is a projection

$$
r_{\sigma}: \prod_{i \in \sigma} X_{i} \times \prod_{i \in[m] \backslash \sigma} A_{i} \longrightarrow \prod_{i \in \sigma \cap \omega} X_{i} \times \prod_{i \in \omega \backslash \sigma} A_{i}
$$

Since $K_{\omega}$ is a full subcomplex, the image of $r_{\sigma}$ belongs to $(\underline{X}, \underline{A})^{K_{\omega}}$. The projections $r_{\sigma}$ patch together to give a retraction $r=\bigcup_{\sigma \in K} r_{\sigma}:(\underline{X}, \underline{A})^{K} \longrightarrow(\underline{X}, \underline{A})^{K_{\omega}}$. The naturality assertion follows from the naturality of inclusions and projections.

Proposition 4.3. Let $K$ be a simplicial complex on the index set $[m]$ and let $v$ be a vertex of $K$. Then there is a homotopy equivalence

$$
\Omega(\underline{X}, \underline{A})^{K} \simeq \Omega X_{v} \times \Omega(\underline{X}, \underline{A})^{K \backslash\{v\}} \times \Omega\left(B_{v}^{K} * Y_{v}\right)
$$

which is natural for inclusions of simplicial complexes $K \longrightarrow K^{\prime}$ on the set $[m]$.

Proof. Consider the homotopy fibration

$$
F_{v}^{K} \longrightarrow(\underline{X}, \underline{A})^{K} \stackrel{f}{\longrightarrow}(\underline{X}, \underline{A})^{K \backslash\{v\}} \times X_{v}
$$

from Lemma 4.1. Observe that $K \backslash\{v\}$ and $\{v\}$ are the full subcomplexes of $K$ on the sets $[m]-\{v\}$ and $\{v\}$ respectively. So by Lemma 4.2 , the maps $s_{1}:(\underline{X}, \underline{A})^{K \backslash\{v\}} \longrightarrow(\underline{X}, \underline{A})^{K}$ and $s_{2}: X_{v} \longrightarrow$ $(\underline{X}, \underline{A})^{K}$ have left inverses $(\underline{X}, \underline{A})^{K} \stackrel{f_{1}}{\longrightarrow}(\underline{X}, \underline{A})^{K \backslash\{v\}}$ and $(\underline{X}, \underline{A})^{K} \stackrel{f_{2}}{\longrightarrow} X_{v}=(\underline{X}, \underline{A})^{\{v\}}$ respectively. Since the vertex sets for $K \backslash\{v\}$ and $\{v\}$ are disjoint, the left inverses have the property that $f_{1} \circ s_{2}$ and $f_{2} \circ s_{1}$ are trivial. Lemma 3.7 cannot be applied immediately since $f$ is usually not an $H$-map, but after looping the homotopy fibration (10) it can be applied, and this gives the asserted homotopy equivalence.

The naturality property follows from the naturality properties of the simplicial map $K \longrightarrow$ $K \backslash\{v\} *\{v\}$, the polyhedral product and Lemma 4.2 , together with the fact that $\Omega(\underline{X}, \underline{A})^{K} \longrightarrow$ $\Omega(\underline{X}, \underline{A})^{K^{\prime}}$ is an $H$-map.

One more preliminary result is needed before the proof of Theorem 1.1. Let $K$ be a simplicial complex on the vertex set $[m]$, let $K^{f}$ be the flagification of $K$, and let $L$ be the simplicial complex 
consisting of the vertices of $K$. Let $M$ be either $L$ or $K$. If $v$ is a vertex of $K$ then the simplicial map $M \longrightarrow K^{f}$ implies that there is commutative diagram of simplicial complexes

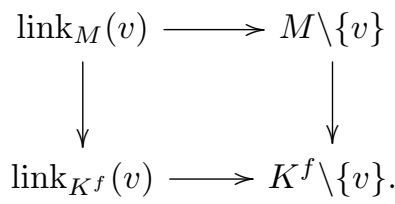

Taking polyhedral products and then taking homotopy fibres gives a homotopy fibration diagram

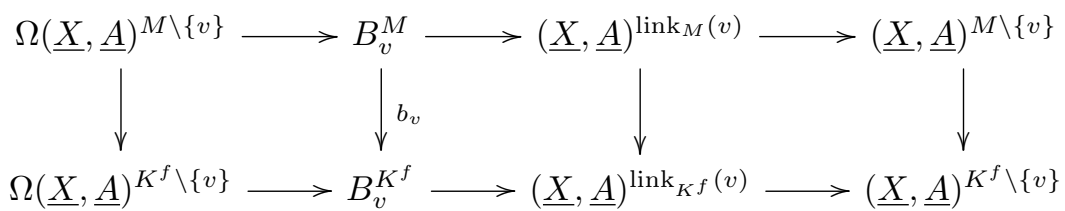

for some induced map of fibres $b_{v}$.

Lemma 4.4. Let $M$ be either $L$ or $K$. Suppose that in (11) the map $\Omega(\underline{X}, \underline{A})^{M \backslash\{v\}} \longrightarrow \Omega(\underline{X}, \underline{A})^{K^{f} \backslash\{v\}}$ has a right homotopy inverse. Then $b_{v}$ has a right homotopy inverse $s_{v}: B_{v}^{K^{f}} \longrightarrow B_{v}^{M}$. Moreover, $s_{v}$ can be chosen so that it factors through the $\operatorname{map} \Omega(\underline{X}, \underline{A})^{M \backslash\{v\}} \longrightarrow B_{v}^{M}$.

Proof. Consider the homotopy fibration along the bottom row of (11). Since $K^{f}$ is flag, by Lemma 2.3, $\operatorname{link}_{K^{f}}(v)$ is a full subcomplex of $K^{f} \backslash\{v\}$. Thus $(\underline{X}, \underline{A})^{\operatorname{link}_{K^{f}}(v)}$ is a retract of $(\underline{X}, \underline{A})^{K^{f} \backslash\{v\}}$. Therefore, by Lemma 3.6, the map $\Omega(\underline{X}, \underline{A})^{K^{f} \backslash\{v\}} \longrightarrow B_{v}^{K^{f}}$ has a right homotopy inverse $t: B_{v}^{K^{f}} \longrightarrow$ $\Omega(\underline{X}, \underline{A})^{K^{f} \backslash\{v\}}$. By hypothesis, the map $\Omega(\underline{X}, \underline{A})^{M \backslash\{v\}} \longrightarrow \Omega(\underline{X}, \underline{A})^{K^{f} \backslash\{v\}}$ has a right homotopy inverse $s: \Omega(\underline{X}, \underline{A})^{K^{f} \backslash\{v\}} \longrightarrow \Omega(\underline{X}, \underline{A})^{M \backslash\{v\}}$. Thus there is a homotopy commutative diagram

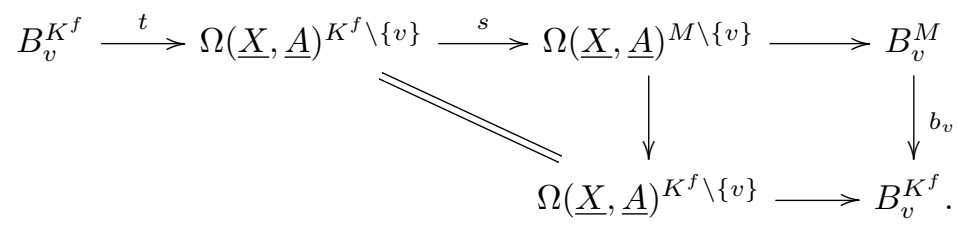

As the lower direction around the diagram is homotopic to the identity map on $B_{v}^{K^{f}}$, the upper direction around the diagram implies that $b_{v}$ has a right homotopy inverse.

Proof of Theorem 1.1. Let $K$ be a simplicial complex on the vertex set $[m]$, let $K^{f}$ be its flagification, and let $L$ be $m$ disjoint points. Then there is a sequence of inclusions of simplicial complexes $L \longrightarrow K \longrightarrow K^{f}$. Taking polyhedral products with respect to $(\underline{X}, \underline{A})$ gives a sequence of maps $h:(\underline{X}, \underline{A})^{L} \stackrel{g}{\longrightarrow}(\underline{X}, \underline{A})^{K} \stackrel{f}{\longrightarrow}(\underline{X}, \underline{A})^{K^{f}}$ We will show that $\Omega h$ has a right homotopy inverse, implying that the map $\Omega f: \Omega(\underline{X}, \underline{A})^{K} \longrightarrow \Omega(\underline{X}, \underline{A})^{K^{f}}$ also has a right homotopy inverse. This would prove both parts of the statement of the theorem.

The proof is by induction on the number of vertices. If $m=1$, then $L, K$ and $K^{f}$ all equal the single vertex $\{1\}$, implying that $h$ is the identity map, and so $\Omega h$ has a right homotopy inverse. Assume that the statement of the theorem holds for all simplicial complexes with strictly less than $m$ 
vertices. The decomposition and naturality statements in Proposition 4.3 imply that there is a homotopy commutative diagram of homotopy equivalences

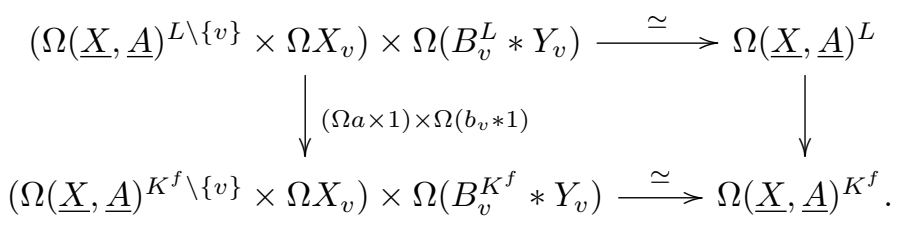

Observe that $K^{f} \backslash\{v\}$ has $m-1$ vertices and $L \backslash\{v\} \longrightarrow K^{f} \backslash\{v\}$ is the inclusion of these vertices. Since $L$ and $K^{f}$ are flag complexes, by Lemma 2.2 so are $L \backslash\{v\}$ and $K^{f} \backslash\{v\}$. Therefore, by inductive hypothesis, the map $\Omega a$ has a right homotopy inverse $s: \Omega(\underline{X}, \underline{A})^{K^{f} \backslash\{v\}} \longrightarrow \Omega(\underline{X}, \underline{A})^{L \backslash\{v\}}$. As $L$ and $K^{f}$ are flag complexes and $\Omega a$ has a right homotopy inverse, by Lemma 4.4 the map $b_{v}$ also has a right homotopy inverse $t: B_{v}^{K^{f}} \longrightarrow B_{v}^{L}$. Therefore $t^{\prime}=\Omega(t * 1)$ is a right homotopy inverse for $\Omega\left(b_{v} * 1\right)$. Putting $s$ and $t^{\prime}$ together we obtain a map

$$
\Omega(\underline{X}, \underline{A})^{K^{f} \backslash\{v\}} \times \Omega X_{v} \times \Omega\left(B_{v}^{K^{f}} * Y_{v}\right) \stackrel{s \times 1 \times t^{\prime}}{\longrightarrow} \Omega(\underline{X}, \underline{A})^{L \backslash\{v\}} \times \Omega X_{v} \times \Omega\left(B_{v}^{L} * Y_{v}\right)
$$

which is a right homotopy inverse of $(\Omega a \times 1) \times \Omega\left(b_{v} * 1\right)$. The homotopy equivalences in (12) therefore imply that the map $h: \Omega(\underline{X}, \underline{A})^{L} \longrightarrow \Omega(\underline{X}, \underline{A})^{K^{f}}$ has a right homotopy inverse. This completes the induction.

\section{ReFinements}

This section gives two refinements describing the homotopy type of the space $B_{v}^{K}$ under certain conditions. First consider the homotopy fibration diagram (11) in the case when $M=K$. Define the space $D_{v}^{K}$ and the map $d_{v}$ by the homotopy fibration

$$
D_{v}^{K} \stackrel{d_{v}}{\longrightarrow} B_{v}^{K} \stackrel{b_{v}}{\longrightarrow} B_{v}^{K^{f}}
$$

Lemma 5.1. Given the hypotheses of Lemma 4.4, there is a homotopy equivalence $B_{v}^{K} \simeq B_{v}^{K^{f}} \times D_{v}^{K}$.

Proof. By Lemma 4.4, $b_{v}$ has a right homotopy inverse $B_{v}^{K^{f}} \stackrel{s_{v}}{\longrightarrow} B_{v}^{K}$. As $B_{v}^{K}$ need not be an $H$-space this does not immediately imply that it is homotopy equivalent to $B_{v}^{K^{f}} \times D_{v}^{K}$. However, Lemma 4.4 also says that $s_{v}$ can be chosen to factor through the homotopy fibration connecting map $\Omega(\underline{X}, \underline{A})^{K \backslash\{v\}} \longrightarrow B_{v}^{K}$. That is, $s_{v}$ can be chosen to be a composite $B_{v}^{K^{f}} \stackrel{s_{v}^{\prime}}{\longrightarrow} \Omega(\underline{X}, \underline{A})^{K \backslash\{v\}} \longrightarrow B_{v}^{K}$ for some map $s_{v}^{\prime}$. For any homotopy fibration sequence $\Omega B \stackrel{\delta}{\longrightarrow} F \longrightarrow E \longrightarrow B$ the connecting map $\delta$ satisfies a homotopy action $\theta: \Omega B \times F \longrightarrow F$ which restricts to the identity map on $F$ and $\delta$ on $\Omega B$. In our case, we obtain a composite

$$
\psi: B_{v}^{K^{f}} \times D_{v}^{K} \stackrel{s_{v}^{\prime} \times d_{v}}{\longrightarrow} \Omega(\underline{X}, \underline{A})^{K \backslash\{v\}} \times B_{v}^{K} \stackrel{\theta}{\longrightarrow} B_{v}^{K} .
$$

Observe that the restriction of $\psi$ to $B_{v}^{K^{f}}$ is $s_{v}$ and the restriction to $D_{v}^{K}$ is $d_{v}$. Thus $\psi$ is a trivialization of the homotopy fibration (13), implying that it is a homotopy equivalence. 
Second, suppose that $K$ is a flag complex. By Lemma $2.3, \operatorname{link}_{K}(v)$ is a full subcomplex of $K \backslash\{v\}$. So by Lemma 4.2 , the inclusion $(\underline{X}, \underline{A})^{\operatorname{link}_{K}(v)} \longrightarrow(\underline{X}, \underline{A})^{K \backslash\{v\}}$ has a left inverse. Define $C_{v}^{K}$ by the homotopy fibration

$$
C_{v}^{K} \longrightarrow(\underline{X}, \underline{A})^{K \backslash\{v\}} \longrightarrow(\underline{X}, \underline{A})^{\operatorname{link}_{K}(v)} .
$$

From the retraction of $(\underline{X}, \underline{A})^{\operatorname{link}_{K}(v)}$ off $(\underline{X}, \underline{A})^{K \backslash\{v\}}$ and the definitions of $B_{v}^{K}$ and $C_{v}^{K}$ we obtain a homotopy pullback diagram

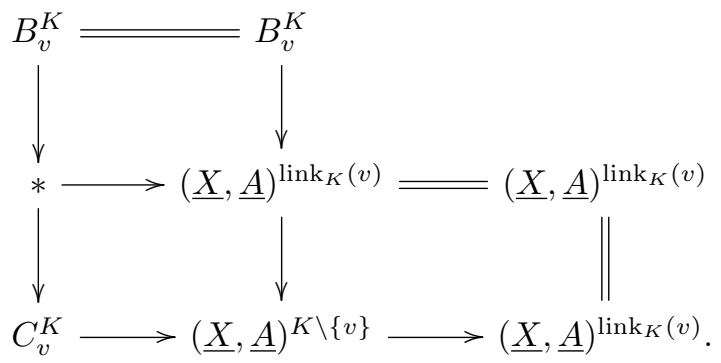

Thus $B_{v}^{K} \simeq \Omega C_{v}^{K}$.

Lemma 5.2. Let $K$ be a flag complex on the vertex set $[m]$ and let $v$ be a vertex of $K$. Then there are homotopy equivalences

$$
\begin{aligned}
& \Omega(\underline{X}, \underline{A})^{K} \simeq \Omega X_{v} \times \Omega(\underline{X}, \underline{A})^{K \backslash\{v\}} \times \Omega\left(\Omega C_{v}^{K} * Y_{v}\right) \\
& \Omega(\underline{X}, \underline{A})^{K \backslash\{v\}} \simeq \Omega(\underline{X}, \underline{A})^{\operatorname{link}_{K}(v)} \times \Omega C_{v}^{K} .
\end{aligned}
$$

Proof. The first homotopy equivalence follows immediately from Proposition 4.3, while the second is an immediate consequence of the homotopy fibration (14) and the retraction of $(\underline{X}, \underline{A})^{\operatorname{link}_{K}(v)}$ off $(\underline{X}, \underline{A})^{K \backslash\{v\}}$.

\section{CO- $H$-SPACE PROPERTIES}

In this section we consider polyhedral products of the form $(\underline{C Y}, \underline{Y})^{K}$ and identify the class of flag complexes $K$ for which $(\underline{C Y}, \underline{Y})^{K}$ is a co- $H$-space. As a corollary, we obtain conditions that allow for a delooping of the statement of Theorem 1.1. This begins with an abstract lemma.

Lemma 6.1. Let $A$ and $B$ be pointed spaces with the homotopy types of $C W$-complexes. Suppose that there is a pointed map $f: A \longrightarrow B$ and $B$ is a co-H-space. If $\Omega f$ has a right homotopy inverse then $f$ has a right homotopy inverse.

Proof. Since $B$ is a co- $H$-space, by [G2] there is a map $s: B \longrightarrow \Sigma \Omega B$ which is a right homotopy inverse to the canonical evaluation map ev: $\Sigma \Omega B \longrightarrow B$. Let $t: \Omega B \longrightarrow \Omega A$ be a right homotopy 
inverse of $\Omega f$. Consider the diagram

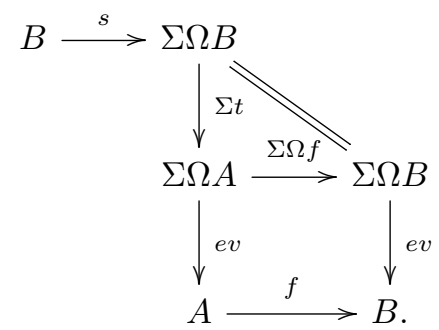

The upper triangle homotopy commutes since $t$ is a right homotopy inverse of $\Omega f$. The lower square homotopy commutes by the naturality of the evaluation map. The upper direction around the diagram is homotopic to $e v \circ s$, which is the identity map on $B$. The lower direction around the diagram therefore implies that $e v \circ \Sigma t \circ s$ is a right homotopy inverse of $f$.

Proposition 6.2. Let $K$ be a simplicial complex on the vertex set $[\mathrm{m}]$, let $K^{f}$ be the flagification of $K$, and let $Y_{1}, \ldots, Y_{m}$ be pointed $C W$-complexes. If $(\underline{C Y}, \underline{Y})^{K^{f}}$ is homotopy equivalent to a co$H$-space then the map $f:(\underline{C Y}, \underline{Y})^{K} \longrightarrow(\underline{C Y}, \underline{Y})^{K^{f}}$ induced by the simplicial inclusion $K \longrightarrow K^{f}$ has a right homotopy inverse.

Proof. Taking $(\underline{X}, \underline{A})=(\underline{C Y}, \underline{Y})$, by Theorem 1.1, $\Omega f: \Omega(\underline{C Y}, \underline{Y})^{K} \longrightarrow \Omega(\underline{C Y}, \underline{Y})^{K^{f}}$ has a right homotopy inverse. Since $(\underline{C Y}, \underline{Y})^{K^{f}}$ is a co-H-space, Lemma 6.1 implies that $f$ has a right homotopy inverse.

Remark 6.3. Note that in Proposition 6.2 we do not need to assume that $Y_{1}, \ldots, Y_{m}$ are pathconnected. Since we asssume that every singleton of $[m]$ is a vertex ( $K$ is on the vertex set $[m])$, $(\underline{C Y}, \underline{Y})^{K}$ is path-connected even if $Y$ is not.

Next we obtain a characterisation of those flag complexes $K$ for which $(\underline{C Y}, \underline{Y})^{K}$ is a co- $H$-space. In terms of notation, when all pairs in the sequence $\left\{\left(X_{i}, A_{i}\right)\right\}_{i=1}^{m}$ are the same, $\left(X_{i}, A_{i}\right)=(X, A)$, we use the notation $(X, A)^{K}$ for $(\underline{X}, \underline{A})^{K}$. Special cases are the Davis-Januskiewicz space $D J(K)=$ $\left(\mathbb{C} P^{\infty}, *\right)^{K}$ and the moment-angle complex $\mathcal{Z}_{K}=\left(D^{2}, S^{1}\right)^{K}$.

A graph $\Gamma$ is called chordal if each of its cycles with $\geq 4$ vertices has a chord (an edge joining two vertices that are not adjacent in the cycle). Equivalently, a chordal graph is a graph with no induced cycles of length more than three. By the result of Fulkerson and Gross [FG] a graph is chordal if and only if its vertices can be ordered in such a way that, for each vertex $i$, the lesser neighbours of $i$ form a clique. Such an order of vertices is called a perfect elimination ordering.

By [GPTW], $\mathcal{Z}_{K^{f}}=\left(D^{2}, S^{1}\right)^{K^{f}}$ is homotopy equivalent to a wedge of spheres if and only if the 1-skeleton of $K^{f}$ is a chordal graph. In particular, if the 1-skeleton of $K^{f}$ is a chordal graph then $\mathcal{Z}_{K^{f}}$ is a co- $H$-space. This result is readily extended to general polyhedral products of the form $(\underline{C Y}, \underline{Y})^{K}$, where $C Y$ denotes the cone over $Y$. Let $X^{\vee k}$ be the $k$-fold wedge of $X$.

Theorem 6.4. Assume that $K$ is a flag complex on the vertex set $[m]$ and $\widetilde{H}^{*}\left(Y_{i} ; \mathbb{Z}\right) \neq 0$ for $1 \leq i \leq m$. The following conditions are equivalent 
(a) the 1-skeleton $K^{1}$ is a chordal graph;

(b) $(\underline{C Y}, \underline{Y})^{K}$ is a co-H-space.

Furthermore, if $K^{1}$ is chordal, there is a homotopy equivalence

$$
(\underline{C Y}, \underline{Y})^{K} \simeq \bigvee_{k=2}^{m} \bigvee_{1 \leq i_{1}<\cdots<i_{k} \leq m}\left(\Sigma Y_{i_{1}} \wedge \cdots \wedge Y_{i_{k}}\right)^{\vee c\left(i_{1}, \ldots, i_{k}\right)}
$$

where $c\left(i_{1}, \ldots, i_{k}\right)=\operatorname{rank} \widetilde{H}^{0}\left(K_{\left\{i_{1}, \ldots, i_{k}\right\}}\right)$ is one less than the number of connected components of the full subcomplex $K_{\left\{i_{1}, \ldots, i_{k}\right\}}$.

Proof. The argument is similar to [GPTW, Theorem 4.6] or [PV, Theorem 4.3], but this time we keep track of the wedge summands. Assume that $K^{1}$ is chordal. Choose a perfect elimination ordering of vertices, and for each vertex $i=1, \ldots, m$ denote by $\sigma_{i}$ the face of $K$ corresponding to the clique of $K^{1}$ consisting of $i$ and its lesser neighbours. All maximal faces of $K$ are among $\sigma_{1}, \ldots, \sigma_{m}$, so we have $\bigcup_{i=1}^{m} \sigma_{i}=K$. Furthermore, for each $k=1, \ldots, m$ the perfect elimination ordering on $K$ induces such an ordering on the full subcomplex $K_{\{1, \ldots, k-1\}}$, so we have $\bigcup_{i=1}^{k-1} \sigma_{i}=K_{\{1, \ldots, k-1\}}$. In particular, the simplicial complex $\bigcup_{i=1}^{k-1} \sigma_{i}$ is flag as a full subcomplex in a flag complex. The intersection $\sigma_{k} \cap \bigcup_{i=1}^{k-1} \sigma_{i}$ is a clique $\sigma_{k} \backslash\{k\}$, so it is a face of $\bigcup_{i=1}^{k-1} \sigma_{i}$. Therefore, $K$ is obtained by iteratively attaching $\sigma_{k}$ to $\bigcup_{i=1}^{k-1} \sigma_{i}$ along the common face $\sigma_{k} \backslash\{k\}$.

We use induction on $m$ to prove the decomposition (15). When $m=1$, both sides of (15) are trivial. Now assume that (15) holds for $K$ with $<m$ vertices. The pushout square (1) for $v=\{m\}$ becomes

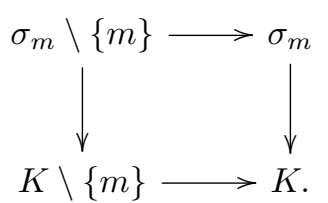

According to our convention, $\sigma_{m} \backslash\{m\}$ and $K \backslash\{m\}$ are regarded as simplicial complexes on $[m] \backslash$ $\{m\}=[m-1]$, while $\sigma_{m}$ is regarded as a complex on $[m]$. The corresponding pushout square (8) of the polyhedral products becomes

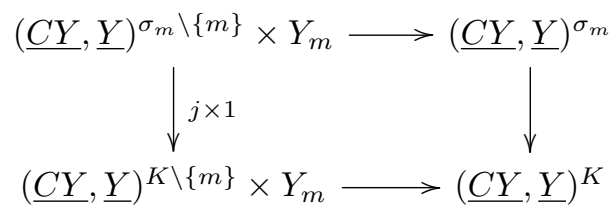

As $\sigma_{m} \backslash\{m\}$ is a face of $K \backslash\{m\}$ and $\sigma_{m}$ is a face of $K$, we have

$$
(\underline{C Y}, \underline{Y})^{\sigma_{m} \backslash\{m\}}=\prod_{i \in \sigma_{m} \backslash\{m\}} C Y_{i} \times \prod_{i \notin \sigma_{m}} Y_{i}, \quad(\underline{C Y}, \underline{Y})^{\sigma_{m}}=\prod_{i \in \sigma_{m}} C Y_{i} \times \prod_{i \notin \sigma_{m}} Y_{i}
$$

Since each $\{i\}$ is a vertex of $K$, the inclusion $\prod_{i \in \omega} Y_{i} \rightarrow(\underline{C Y}, \underline{Y})^{K}$ is null-homotopic for any subset $\omega \subseteq[m]$, and the same holds with $K$ replaced by $K \backslash\{m\}$. Hence, the map $j \times 1$ in (16) decomposes 
into the composition $i_{2} \circ \pi_{2}$ of the projection onto the second factor and the inclusion. It follows that the pushout square (16) decomposes as

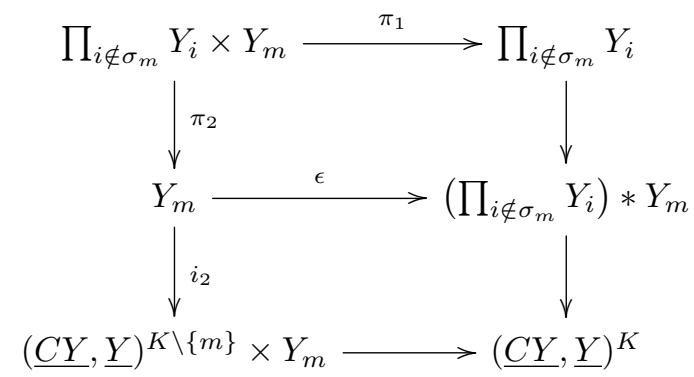

where the map $\epsilon$ is null-homotopic. From the bottom pushout square we obtain

$$
(\underline{C Y}, \underline{Y})^{K} \simeq\left((\underline{C Y}, \underline{Y})^{K \backslash\{m\}} \rtimes Y_{m}\right) \vee\left(\left(\prod_{i \notin \sigma_{m}} Y_{i}\right) * Y_{m}\right),
$$

where $X \rtimes Y=X \times Y /(* \times Y)$ is the right half-smash product, which is homotopy equivalent to $X \vee(X \wedge Y)$ when $X$ is a suspension. By the inductive hypothesis, $(\underline{C Y}, \underline{Y})^{K \backslash\{m\}}$ is a suspension, so we can rewrite the identity above as

$$
(\underline{C Y}, \underline{Y})^{K} \simeq(\underline{C Y}, \underline{Y})^{K \backslash\{m\}} \vee\left((\underline{C Y}, \underline{Y})^{K \backslash\{m\}} \wedge Y_{m}\right) \vee\left(\underset{\substack{1 \leq i_{1}<\cdots<i_{k} \leq m-1 \\\left\{i_{j}, m\right\} \notin K}}{\bigvee} \Sigma Y_{i_{1}} \wedge \cdots \wedge Y_{i_{k}} \wedge Y_{m}\right)
$$

Now a simple counting argument together with the inductive hypothesis gives (15). This also proves the implication $(\mathrm{a}) \Rightarrow(\mathrm{b})$.

To prove the implication (b) $\Rightarrow(\mathrm{a})$, assume that $K^{1}$ is not chordal. Choose an induced chordless cycle $K_{\omega}$ with $|\omega| \geq 4$ (i. e. a full subcomplex isomorphic to the boundary of an $|\omega|$-gon). Then there is a nontrivial product in the cohomology ring $H^{*}\left((\underline{C Y}, \underline{Y})^{K_{\omega}} ; \mathbb{Z}\right)$. (When $(\underline{C Y}, \underline{Y})=\left(D^{1}, S^{0}\right)$, the polyhedral product $\left(D^{1}, S^{0}\right)^{K_{\omega}}$ is an orientable surface of positive genus [BP1, Example 6.40]; the general case then follows from [BBCG2, Theorem 1.9]). By Lemma 4.2, the same nontrivial product appears in $H^{*}\left((\underline{C Y}, \underline{Y})^{K} ; \mathbb{Z}\right)$. Thus, $(\underline{C Y}, \underline{Y})^{K}$ is not a co- $H$-space.

Remark 6.5. Theorem 6.4 implies that the wedge decomposition of $\Sigma(\underline{C Y}, \underline{Y})^{K}$ of [BBCG1] desuspends when $K$ is flag and $K^{1}$ is chordal; this also follows from the results of Iriye and Kishimoto [IK, Theorem 1.2, Proposition 3.2]. Other classes of simplicial complexes $K$ with this property are described in $[\mathrm{IK}]$ and [GT3]. The novelty of Theorem 6.4 compared to [IK] is the description of the wedge decomposition of $(\underline{C Y}, \underline{Y})^{K}$ in terms of the degree zero cohomology of full subcomplexes of $K$, which does not follow readily from desuspending the decomposition in [BBCG1].

When $K$ is not flag, the implication (b) $\Rightarrow(\mathrm{a})$ of Theorem 6.4 still holds, but (a) $\Rightarrow(\mathrm{b})$ fails. Indeed one can take $K$ to be the boundary of a cyclic polytope [BP2, Example 1.1.17] of dimension $n \geq 4$ with $m>n+1$ vertices. Then $K^{1}$ is a complete graph on $m$ vertices, so it is chordal. On the other hand, $\mathcal{Z}_{K}=\left(D^{2}, S^{1}\right)^{K}$ is an $(m+n)$-manifold with nontrivial cohomology product, so it cannot be a co- $H$-space. 
Finally, we give conditions that allow for a delooping of the maps in Theorem 1.1.

Corollary 6.6. Let $K$ be a simplicial complex on the vertex set $[m]$ whose 1-skeleton is a chordal graph. If $K^{f}$ is the flagification of $K$ then the map $f:(\underline{C Y}, \underline{Y})^{K} \longrightarrow(\underline{C Y}, \underline{Y})^{K^{f}}$ has a right homotopy inverse.

Proof. As $K$ and $K^{f}$ have the same 1-skeleton, Theorem 6.4 implies that $(\underline{C Y}, \underline{Y})^{K^{f}}$ is a co-H-space (and even a suspension). The result follows from Proposition 6.2.

Corollary 6.7. Let $K$ be a flag simplicial complex on the vertex set $[m]$, and let $L$ be the simplicial complex given by $m$ disjoint points. The map $h:(\underline{C Y}, \underline{Y})^{L} \longrightarrow(\underline{C Y}, \underline{Y})^{K}$ has a right homotopy inverse if and only if the 1-skeleton of $K$ is a chordal graph.

Proof. Assume that $K^{1}$ is a chordal graph. As $K$ is flag, Theorem 1.1 implies that $\Omega h$ has a right homotopy inverse, and Theorem 6.4 implies that $(\underline{C Y}, \underline{Y})^{K}$ is a co- $H$-space. Then $h$ has a right homotopy inverse by Lemma 6.1 .

Now assume that $h$ has a right homotopy inverse. Then $(\underline{C Y}, \underline{Y})^{K}$ is a co- $H$-space, being a retract of the co- $H$-space $(\underline{C Y}, \underline{Y})^{L}$. Theorem 6.4 implies that $K^{1}$ is a chordal graph.

Remark 6.8. Given $(\underline{C Y}, \underline{Y})^{L} \stackrel{g}{\longrightarrow}(\underline{C Y}, \underline{Y})^{K} \stackrel{f}{\longrightarrow}(\underline{C Y}, \underline{Y})^{K^{f}}$, Theorem 1.1 states that each of the two maps $\Omega f$ and $\Omega h=\Omega f \circ \Omega g$ has a right homotopy inverse. Corollary 6.6 gives a sufficient condition for a delooping of the first map, and Corollary 6.7 gives a necessary and sufficient condition for a delooping of the second map. In both cases the condition is that $K^{1}$ is a chordal graph. However, this condition is obviously not necessary for a delooping of $\Omega f$. Indeed, $f$ has a right inverse for any flag $K$, not only for those with chordal $K^{1}$, because in this case $K^{f}=K$ and $f$ is the identity map.

\section{WHITEHEAD PRODUCTS}

In this section we describe two ways of relating the results of Theorem 1.1 and Theorem 6.4 to the classical iterated Whitehead products. First, we consider polyhedral products of the form $(\underline{X}, \underline{*})^{K}$ with flag $K$ whose 1-skeleton is a chordal graph, and obtain a generalisation (Proposition 7.1) of Porter's description of the homotopy fiber of the inclusion of an $m$-fold wedge into a product in terms of Whitehead brackets. Second, we consider the loop space $\Omega(\underline{S}, \underline{*})^{K}$ on a polyhedral product of spheres for an arbitrary flag complex $K$, and obtain a generalisation (Proposition 7.2) of the Hilton-Milnor Theorem.

First, specialize to the case when each pair $\left(X_{i}, A_{i}\right)$ is of the form $\left(X_{i}, *\right)$ and write $(\underline{X}, \underline{*})$ for $(\underline{X}, \underline{A})$. By $[\mathrm{GT} 1]$, for example, there is a homotopy fibration

$$
(\underline{C \Omega X}, \underline{\Omega X})^{K} \stackrel{\gamma_{K}}{\longrightarrow}(\underline{X}, \underline{*})^{K} \longrightarrow \prod_{i=1}^{m} X_{i}
$$

for any simplicial complex $K$. This is natural for simplicial inclusions, so if $K$ is a flag complex on the vertex set $[m]$ and $L \longrightarrow K$ is the inclusion of the vertex set then there is a homotopy fibration 
diagram

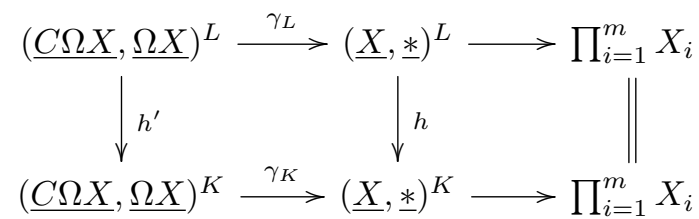

where both $h$ and $h^{\prime}$ are induced maps of polyhedral products. By Theorem 1.1, $\Omega h^{\prime}$ has a right homotopy inverse. Further, if $K^{1}$ is a chordal graph then Proposition 6.2 and Theorem 6.4 imply that $h^{\prime}$ has a right homotopy inverse.

Observe that as $L$ is $m$ disjoint points we have $(\underline{X}, \underline{*})^{L}=X_{1} \vee \cdots \vee X_{m}$, implying that $(\underline{C \Omega X}, \underline{\Omega X})^{L}$ is the homotopy fibre of the inclusion of the wedge into the product. Porter $[\mathrm{P}]$ identified the homotopy type of this fibre, from which we obtain a homotopy equivalence

$$
(\underline{C \Omega X}, \underline{\Omega X})^{L} \simeq \bigvee_{k=2}^{m} \bigvee_{1 \leq i_{1}<\cdots<i_{k} \leq m}\left(\Sigma \Omega X_{i_{1}} \wedge \cdots \wedge \Omega X_{i_{k}}\right)^{\vee(k-1)}
$$

Notice that $L^{1}$ is a chordal graph and the decomposition in (18) exactly matches that of $(\underline{C \Omega X}, \underline{\Omega X})^{L}$ in (15). Moreover, by [T, Theorem 6.2], Porter's homotopy type identification can be chosen so that the composite

$$
\varphi_{L}: \bigvee_{k=2}^{m} \bigvee_{1 \leq i_{1}<\cdots<i_{k} \leq m}\left(\Sigma \Omega X_{i_{1}} \wedge \cdots \wedge \Omega X_{i_{k}}\right)^{\vee(k-1)} \stackrel{\simeq}{\longrightarrow}(\underline{C \Omega X}, \underline{\Omega X})^{L} \stackrel{\gamma_{L}}{\longrightarrow}(\underline{X}, \underline{*})^{L}
$$

is a wedge sum of iterated Whitehead products of the maps

$$
e v_{i}: \Sigma \Omega X_{i} \stackrel{e v}{\longrightarrow} X_{i} \hookrightarrow X_{1} \vee \cdots \vee X_{m}=(\underline{X}, \underline{*})^{L} .
$$

Returning to (17), the naturality of the Whitehead product implies that $h \circ \varphi_{L}$ is a wedge sum of Whitehead products mapping into $(\underline{X}, \underline{*})^{K}$. The right homotopy inverse for $h^{\prime}$ when $K^{1}$ is a chordal graph therefore implies the following.

Proposition 7.1. Let $K$ be a flag complex such that $K^{1}$ is a chordal graph. Then the map $(\underline{C \Omega X}, \underline{\Omega X})^{K} \stackrel{\gamma_{K}}{\longrightarrow}(\underline{X}, \underline{*})^{K}$ factors through a wedge sum of Whitehead products.

In the case when $(\underline{X}, \underline{*})^{K}=\left(\mathbb{C} P^{\infty}, *\right)^{K}=D J(K)$ and $(\underline{C \Omega X}, \underline{\Omega X})^{K} \simeq\left(D^{2}, S^{1}\right)^{K}=\mathcal{Z}_{K}$ the result above follows from [GPTW, Theorem 4.3], where the Whitehead products were explicitly specified as iterated brackets of the canonical generators.

Theorem 1.1 also leads to a generalization of the Hilton-Milnor Theorem. In this case we specialize to pairs $\left(\Sigma X_{i}, *\right)$, giving $(\underline{\Sigma X}, \underline{*})^{L}=\Sigma X_{1} \vee \cdots \vee \Sigma X_{m}$. The Hilton-Milnor Theorem states that there is a homotopy equivalence

$$
\Omega\left(\Sigma X_{1} \vee \cdots \vee \Sigma X_{m}\right) \simeq \prod_{\alpha \in L\langle V\rangle} \Omega\left(\Sigma X_{1}^{\wedge \alpha_{1}} \wedge \cdots \wedge X_{m}^{\wedge \alpha_{m}}\right)
$$

where: $V$ is a free $\mathbb{Z}$-module on $m$ elements $x_{1}, \ldots, x_{m} ; L\langle V\rangle$ is the free Lie algebra on $V ; \alpha$ runs over a $\mathbb{Z}$-module basis of $L\langle V\rangle$; and $\alpha_{i}$ is the number of occurances of $x_{i}$ in the bracket $\alpha$. Here, if 
$\alpha_{i}=0$ we interpret $X_{i}$ as being omitted from the smash product rather than as being trivial. For example, $X_{1}^{\wedge 2} \wedge X_{2}^{0}=X_{1}^{\wedge 2}$. The Hilton-Milnor Theorem also describes the maps from the factors on the right side of (19) into $\Omega\left(\Sigma X_{1} \vee \cdots \vee \Sigma X_{m}\right)$. If the length of $\alpha$ is 1 then the relevant factor is $\Omega \Sigma X_{i}$ for some $i$ and the map $\Omega \Sigma X_{i} \longrightarrow \Omega\left(\Sigma X_{1} \vee \cdots \vee \Sigma X_{m}\right)$ is the loops on the inclusion into the wedge. If the length of $\alpha$ is larger than 1 then the map $\Omega\left(\Sigma X_{1}^{\wedge \alpha_{1}} \wedge \cdots \wedge X_{m}^{\wedge \alpha_{m}}\right) \longrightarrow \Omega\left(\Sigma X_{1} \vee \cdots \vee \Sigma X_{m}\right)$ is the loops on the Whitehead product corresponding to the bracket $\alpha$.

By Theorem 1.1, if $K$ is a flag complex on the vertex set $[m]$ then the map $\Omega(\underline{\Sigma X}, \underline{*})^{L} \stackrel{h}{\longrightarrow}$ $\Omega(\underline{\Sigma X}, \underline{*})^{K}$ has a right homotopy inverse. In particular, $\Omega(\underline{\Sigma X}, \underline{*})^{K}$ is a retract of the product on the right side of (19). It is probably the case that the retraction consists of selecting an appropriate subproduct, but this is not immediately clear. That is, simply knowing that $\Omega h$ has a right homotopy inverse leaves open the possibility that some of the factors $\Omega\left(\Sigma X_{1}^{\wedge \alpha_{1}} \wedge \cdots \wedge X_{m}^{\wedge \alpha_{m}}\right)$ split as $A \times B$ where $A$ retracts off $\Omega(\underline{\Sigma X}, \underline{*})^{K}$ while $B$ does not. However, if we specialize a bit more then this possibility is essentially eliminated.

Suppose that each $X_{i}$ is a connected sphere $S^{n_{i}-1}$ and write $(\underline{S}, \underline{*})$ for $(\underline{\Sigma X}, \underline{*})$. Since each $X_{i}$ is a sphere, the space $\Sigma X_{1}^{\wedge \alpha_{1}} \wedge \cdots \wedge X_{m}^{\wedge \alpha_{m}}$ is homotopy equivalent to a sphere, so the right side of (19) becomes a product of looped spheres. The space $\Omega S^{n}$ is indecomposable unless $n \in\{2,4,8\}$. In the latter case, we have a homotopy equivalence $\Omega H \times E: \Omega S^{2 n-1} \times S^{n-1} \stackrel{\simeq}{\longrightarrow} \Omega S^{n}$, which is a product of the looped Hopf map $H$ and the suspension map $E$. The retraction of $\Omega(\underline{S}, \underline{*})^{K}$ off $\Omega(\underline{S}, \underline{*})^{L}$ implies the following.

Proposition 7.2. Let $K$ be a flag complex. Then

$$
\Omega(\underline{S}, \underline{*})^{K} \simeq\left(\prod_{i=1}^{m} \Omega S^{n_{i}}\right) \times M
$$

where $M$ is homotopy equivalent to a product of spheres and loops on spheres. Further,

(a) a factor $\Omega S^{n}$ of $M$ with $n \notin\{3,7,15\}$ maps to $\Omega(\underline{S} \text {, })^{K}$ by a looped Whitehead product $\Omega S^{n} \stackrel{\Omega w}{\longrightarrow} \Omega(\underline{S}, \underline{*})^{K}$

(b) a factor $\Omega S^{2 n-1}$ of $M$ with $n \in\{2,4,8\}$ maps to $\Omega(\underline{S}, \underline{*})^{K}$ by a looped Whitehead product $\Omega S^{2 n-1} \stackrel{\Omega w}{\longrightarrow} \Omega(\underline{S}, \underline{*})^{K}$ or by a composite $\Omega S^{2 n-1} \stackrel{\Omega H}{\longrightarrow} \Omega S^{n} \stackrel{\Omega w}{\longrightarrow} \Omega(\underline{S}, \underline{*})^{K}$, where $H$ is the Hopf map;

(c) a factor $S^{n-1}$ of $M$ has $n \in\{2,4,8\}$ and maps to $\Omega(\underline{S}, \underline{*})^{K}$ by a composite $S^{n-1} \stackrel{E}{\longrightarrow}$ $\Omega S^{n} \stackrel{\Omega w}{\longrightarrow} \Omega(\underline{S}, \underline{*})^{K}$, where $E$ is the suspension map and $w$ is a Whitehead product.

Refining a bit, by [GT1] the homotopy fibration $(\underline{C \Omega S}, \underline{\Omega S})^{K} \stackrel{\gamma_{K}}{\longrightarrow}(\underline{S}, \underline{*})^{K} \longrightarrow \prod_{i=1}^{m} S^{n_{i}}$ splits after looping to give a homotopy equivalence

$$
\Omega(\underline{S}, \underline{*})^{K} \simeq\left(\prod_{i=1}^{m} \Omega S^{n_{i}}\right) \times \Omega(\underline{C \Omega S}, \underline{\Omega S})^{K} .
$$


Therefore, Proposition 7.2 implies that if $K$ is a flag complex then $\Omega(\underline{C \Omega S}, \underline{\Omega S})^{K}$ is homotopy equivalent to a product of spheres and loops on spheres, and under this homotopy equivalence $\Omega \gamma_{K}$ becomes a product of maps of the from $\Omega w, \Omega w \circ \Omega H$ or $\Omega w \circ E$.

This has implications for moment-angle complexes and Davis-Januszkiewicz spaces. Recall that $D J(K) \simeq\left(\mathbb{C} P^{\infty}, *\right)^{K}$ and $\mathcal{Z}_{K} \simeq\left(D^{2}, S^{1}\right)^{K}$. There is a homotopy fibration

$$
\mathcal{Z}_{K} \stackrel{\psi_{K}}{\longrightarrow} D J(K) \longrightarrow \prod_{i=1}^{m} \mathbb{C} P^{\infty}
$$

which splits after looping to give a homotopy equivalence

$$
\Omega D J(K) \simeq\left(\prod_{i=1}^{m} S^{1}\right) \times \Omega \mathcal{Z}_{K}
$$

The inclusion $S^{2} \longrightarrow \mathbb{C} P^{\infty}$ induces maps of pairs $\left(S^{2}, *\right) \longrightarrow\left(\mathbb{C} P^{\infty}, *\right)$ and $\left(C \Omega S^{2}, \Omega S^{2}\right) \longrightarrow$ $\left(C \Omega \mathbb{C} P^{\infty}, \Omega \mathbb{C} P^{\infty}\right) \stackrel{\simeq}{\longrightarrow}\left(D^{2}, S^{1}\right)$. These then induce a commutative diagram of polyhedral products

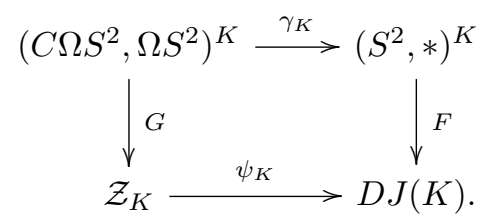

Observe that the suspension map $S^{1} \stackrel{E}{\longrightarrow} \Omega S^{2}$ induces a map of pairs $\left(C S^{1}, S^{1}\right) \longrightarrow\left(C \Omega S^{2}, \Omega S^{2}\right)$ with the property that the composite $\left(C S^{1}, S^{1}\right) \longrightarrow\left(C \Omega S^{2}, \Omega S^{2}\right) \longrightarrow\left(D^{2}, S^{1}\right)$ is a homotopy equivalence. This implies that the map $G$ in (20) has a right homotopy inverse. If $K$ is a flag complex then Proposition 7.2 says that $\Omega\left(C \Omega S^{2}, \Omega S^{2}\right)^{K}$ is homotopy equivalent to a product of spheres and loops on spheres, and the factors map to $\Omega\left(S^{2}, *\right)^{K}$ by maps of the form $\Omega w, \Omega w \circ \Omega H$ or $\Omega w \circ E$. Thus from the map $G$ in (20) having a right homotopy inverse, and $F$ being natural with respect to Whitehead products, we obtain the following.

Corollary 7.3. Let $K$ be a flag complex. Then $\Omega \mathcal{Z}_{K}$ is homotopy equivalent to a product of spheres and loops on spheres, and under this equivalence the map $\Omega \mathcal{Z}_{K} \stackrel{\Omega \psi_{K}}{\longrightarrow} \Omega D J(K)$ becomes a product of maps of the form $\Omega w, \Omega w \circ \Omega H$ or $\Omega w \circ E$ where $w$ is a Whitehead product.

Notice that $\mathcal{Z}_{K}$ itself is often not a product or a wedge of spheres. For example, if $K$ is the boundary of an $n$-gon for $n \geq 5$ then $K$ is flag and $\mathcal{Z}_{K}$ is diffeomorphic to a connected sum of products of two spheres. Nevertheless, $\Omega \mathcal{Z}_{K}$ is homotopy equivalent to a product of spheres and loops on spheres.

\section{Homotopy theOREtic CONSEQUenCES}

We restrict attention to Davis-Januszkiewicz spaces $D J(K)=\left(\mathbb{C} P^{\infty}, *\right)^{K}$ and moment-angle complexes $\mathcal{Z}_{K}=\left(D^{2}, S^{1}\right)^{K}$. Let $S^{2} \longrightarrow \mathbb{C} P^{\infty}$ be the inclusion of $S^{2} \cong \mathbb{C} P^{1}$ into $\mathbb{C} P^{\infty}$. Then there is an induced map of polyhedral products

$$
i_{K}:\left(S^{2}, *\right)^{K} \longrightarrow\left(\mathbb{C} P^{\infty}, *\right)^{K} .
$$


Building on the fact that the map $G$ in (20) has a right homotopy inverse, in [GT3] the following was proved.

Lemma 8.1. The map $\Omega i_{K}$ has a right homotopy inverse.

Lemma 8.2. Let $K$ be a flag complex. Suppose that there is a map $h:\left(\mathbb{C} P^{\infty}, *\right)^{K} \longrightarrow Y$ where $Y$ is 2-connected. Then $\Omega h$ is null homotopic. Consequently, $h$ induces the zero map on homotopy groups.

Proof. Let $L$ be the simplicial complex on $m$ disjoint points. The simplicial map $L \longrightarrow K$ induces a map of polyhedral products $g:\left(S^{2}, *\right)^{L} \longrightarrow\left(S^{2}, *\right)^{K}$. Consider the composite

$$
\left(S^{2}, *\right)^{L} \stackrel{g}{\longrightarrow}\left(S^{2}, *\right)^{K} \stackrel{i_{K}}{\longrightarrow}\left(\mathbb{C} P^{\infty}, *\right)^{K} \stackrel{h}{\longrightarrow} Y .
$$

Observe that by the definition of the polyhedral product, $\left(S^{2}, *\right)^{L} \simeq \bigvee_{i=1}^{m} S^{2}$. Since $Y$ is 2-connected, the composite $h \circ i_{K} \circ g$ is therefore null homotopic. Since $K$ is a flag complex, by Theorem 1.1, $\Omega g$ has a right homotopy inverse. Therefore $\Omega h \circ \Omega i_{K}$ is null homotopic. By Lemma 8.1, $\Omega i_{K}$ also has a right homotopy inverse. Therefore $\Omega h$ is null homotopic.

For example, let $C$ be the homotopy cofibre of the composite

$$
\psi: \bigvee_{i=1}^{m} S^{2} \longrightarrow \bigvee_{i=1}^{m} \mathbb{C} P^{\infty} \longrightarrow D J(K)
$$

where the left map is the wedge of inclusions of the bottom cells and the right map is the map of polyhedral products induced by including the vertices into $K$. The description of $H^{*}(D J(K) ; \mathbb{Z})$ (see, for example [BP1]) implies that $C$ is 3-connected. Therefore Lemma 8.2 implies that if $K$ is a flag complex then the quotient map

$$
f: D J(K) \longrightarrow C=D J(K) /\left(\bigvee_{i=1}^{m} S^{2}\right)
$$

induces the trivial map on homotopy groups.

Lemma 8.2 says that if $K$ is a flag complex then the bottom 2-spheres in $D J(K)$ have a great impact on its homotopy theory. The next lemma says this much more dramatically in the case of $\mathcal{Z}_{K}$ when $K^{1}$ is a chordal graph.

Lemma 8.3. Let $K$ be a flag complex such that $K^{1}$ is a chordal graph. Then there is a homotopy commutative diagram

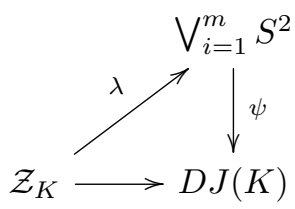

for some map $\lambda$. 
Proof. As usual, let $L$ be the vertex set of $K$. Consider the diagram

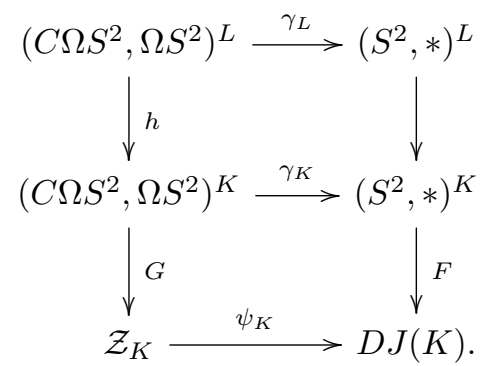

The upper square is induced by the simplicial inclusion of $L$ into $K$. The lower square homotopy commutes by (20). Notice that the right column is equal to $\psi$. As mentioned in the previous section, the map $G$ has a right homotopy inverse. Since $K$ is a flag complex and $K^{1}$ is a chordal graph, by Corollary 6.7, the map $h$ has a right homotopy inverse. Thus $G \circ h$ has a right homotopy inverse and the lemma follows.

\section{REFERENCES}

[BBCG1] A. Bahri, M. Bendersky, F.R. Cohen, and S. Gitler, The polyhedral product functor: a method of decomposition for moment-angle complexes, arrangements and related spaces, Adv. Math. 225 (2010), 1634-1668.

[BBCG2] A. Bahri, M. Bendersky, F.R. Cohen, and S. Gitler, Cup-products for the polyhedral product functor, Math. Proc. Camb. Phil. Soc. 153 (2012), 457-469.

[BP1] V.M. Buchstaber and T.E. Panov, Torus actions and their applications in topology and combinatorics, University Lecture Series 24, American Mathematical Society, 2002.

[BP2] V.M. Buchstaber and T.E. Panov, Toric topology, Mathematical Surveys and Monographs 204, American Mathematical Society, 2015.

[DJ] M. W. Davis and T. Januszkiewicz, Convex polytopes, Coxeter orbifolds and torus actions, Duke Mathematical Journal 62 (1991), 417-452.

[FG] D. R. Fulkerson and O. Gross, Incidence matrices and interval graphs, Pacific J. Math 15 (1965), 835-855.

[FT] Y. Félix and D. Tanré, Rational homotopy of the polyhedral product functor, Proc. Amer. Math. Soc. 137 (2009), 891-898.

[G1] T. Ganea, A generalization of the homology and homotopy suspension, Comment. Math. Helv. 39 (1965), 295-322.

[G2] T. Ganea, Cogroups and suspensions, Invent. Math. 9 (1970), 185-197.

[GPTW] J. Grbić, T. Panov, S. Theriault and J. Wu. The homotopy types of moment-angle complexes for flag complexes, Trans. Amer. Math. Soc. 368 (2016), 6663-6682.

[GT1] J. Grbić and S. Theriault, The homotopy type of the complement of a coordinate subspace arrangement, Topology 46 (2007), 357-396.

[GT2] J. Grbić and S. Theriault, The homotopy type of the polyhedral product for shifted complexes, Adv. Math. 245 (2013), 690-715.

[GT3] J. Grbić and S. Theriault, Homotopy theory in toric topology, Russian Math. Surveys 71 (2016), 185-251.

[GW] V. Grujić and V. Welker, Moment-angle complexes of pairs $\left(D^{n}, S^{n-1}\right)$ and simplicial complexes with vertexdecomposable duals, Monatsh. Math. 176 (2015), 255-273.

[IK] K. Iriye and D. Kishimoto, Fat wedge filtration and decomposition of polyhedral products, arXiv:1412.4866, to appear in Kyoto J. Math.

[Ma] M. Mather, Pull-backs in homotopy theory, Canad. J. Math. 28 (1976), 225-263. 
[Mi] J. Milnor, On spaces having the homotopy type of a CW-complex, Trans. Amer. Math. Soc. 90 (1959), $272-280$.

[PV] T. Panov and Ya. Veryovkin, Polyhedral products and commutator subgroups of right-angled Artin and Coxeter groups, Mat. Sbornik 207 (2016), no. 11, 105-126 (Russian); Sbornik Math. 207 (2016), no. 11, 1582-1600 (English translation).

[P] G.J. Porter, The homotopy groups of wedges of suspensions, Amer. J. Math. 88 (1966), 655-663.

[Se] P. Selick, Introduction to Homotopy Theory, Fields Institute Monographs 9, American Mathematical Society, Providence RI, 1997.

[Sp] E.H. Spanier, Algebraic Topology, McGraw-Hill Book Co., New York-Toronto-London, 1966.

[T] S. Theriault, The dual polyhedral product, cocategory and nilpotence, arXiv:1506.05998.

Department of Mathematics and Mechanics, Moscow State University, Leninskie Gory, 119991 Moscow, Russia,

Institute for Theoretical and Experimental Physics, Moscow, Russia and

Institute for Information Transmission Problems, Russian Academy of Sciences

E-mail address: tpanov@mech.math.msu.su

Mathematical Sciences, University of Southampton, Southampton SO17 1BJ, United Kingdom

E-mail address: S.D.Theriault@soton.ac.uk 